\title{
Registro notable de Ateles hybridus en un fragmento de bosque húmedo del municipio de Barrancabermeja, Santander, Colombia
}

\author{
Sebastián Mejía-Correa \\ Grupo Consultor Ingeniería Strycon S.A.S. sebasmeco@gmail.com
}

La Marimonda del Magdalena Ateles hybridus (I. Geoffroy, 1829) es uno de los primates más amenazados de Colombia, considerado en peligro crítico a nivel nacional (MADS, 2014) y global (Urbani et al. 2008) debido a la reducción en su población, como consecuencia de la disminución en la calidad de su hábitat, siendo esta la amenaza más grande que enfrenta (Defler et al. 2006). Asimismo, desde 2004 se encuentra listada entre los 25 primates más amenazados del mundo (Mittermeier et al. 2012). El estudio reciente de Link et al. (2013) documenta cómo la pérdida de hábitat no solamente sigue ocurriendo sino que también se ha intensificado en los últimos 20 años, estimando una destrucción de más del 27\% del área disponible para la especie. La pérdida de su hábitat es aún más evidente en toda la zona del Magdalena medio donde los fragmentos de bosque están desapareciendo. Aunque existen actualmente registros de la especie en bosques fragmentados en el departamento de Santander (Link et al. 2013), no se tenían evidencias confirmadas de la especie en el municipio de Barrancabermeja. Aquí se reportan los primeros registros fotográficos de la especie para este municipio.

Los registros fueron obtenidos en agosto de 2015, durante la caracterización del componente faunístico para el Estudio de Impacto ambiental del proyecto GALA1 realizado por el Grupo Consultor Ingeniería Strycon S.A.S. para la empresa Ecopetrol S.A. en el marco de los estudios ambientales para sus proyectos exploratorios. El sitio de los registros correspondió a un fragmento de bosque abierto de tierra firme, en cercanías al Río Sogamoso, ubicado en la finca La Carolina, vereda El Porvenir del municipio de Barrancabermeja, a $14 \mathrm{~km}$ hacia el norte del casco urbano de la ciudad de Barrancabermeja (Figura 1).

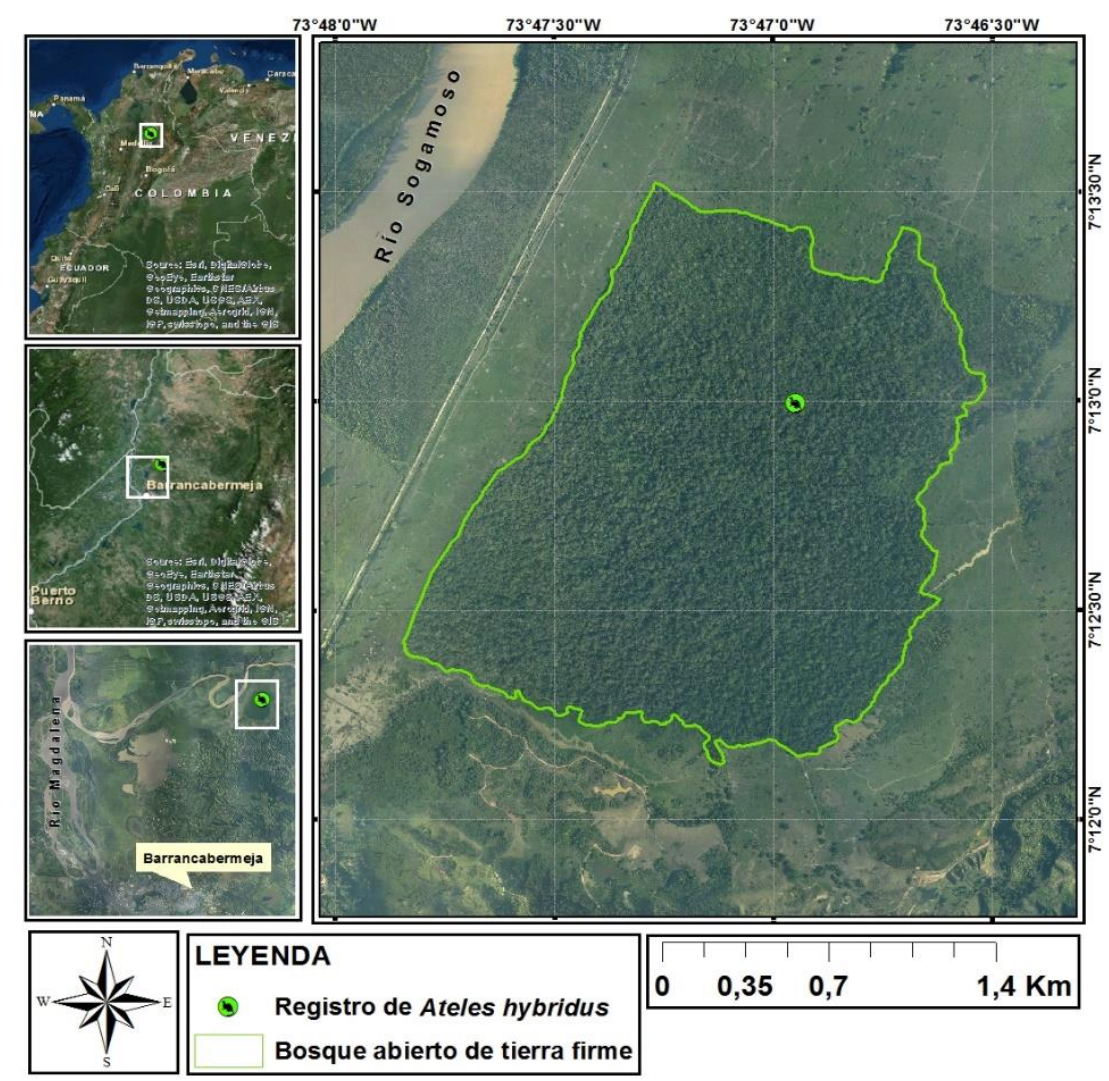

Figura 1. Localidad de registro de Ateles hybridus en un fragmento de bosque del municipio de Barrancabermeja, Santander, Colombia 
Los registros de A. hybridus se lograron durante la realización de recorridos de observación al interior del bosque, el día 13 de agosto de 2015 a las $08 \mathrm{~h} 30$, donde se observó un grupo de seis individuos desplazándose y vocalizando por el dosel del bosque ( $7^{\circ} 12^{\prime} 59.76^{\prime \prime} \mathrm{N}, 73^{\circ} 46^{\prime} 56.89^{\prime \prime} \mathrm{W}, 83 \mathrm{msnm}$ ) (Figura 2). Los individuos observados realizaron algunas vocalizaciones de alerta como respuesta a la presencia de los observadores. Posteriormente, se detuvieron en actitud de exploración por un periodo de 10 minutos, siendo los juveniles los más curiosos y pasivos. Se hicieron dos visitas más a este bosque durante la fase de campo del estudio de Impacto Ambiental (15 y 20 de agosto), observando nuevamente algunos individuos de $A$. hybridus en actividad de forrajeo y observación, donde se pudo constatar el consumo del fruto del hobo, Spondias mombin (Anacardiaceae), especie frecuente y abundante en ésta zona.

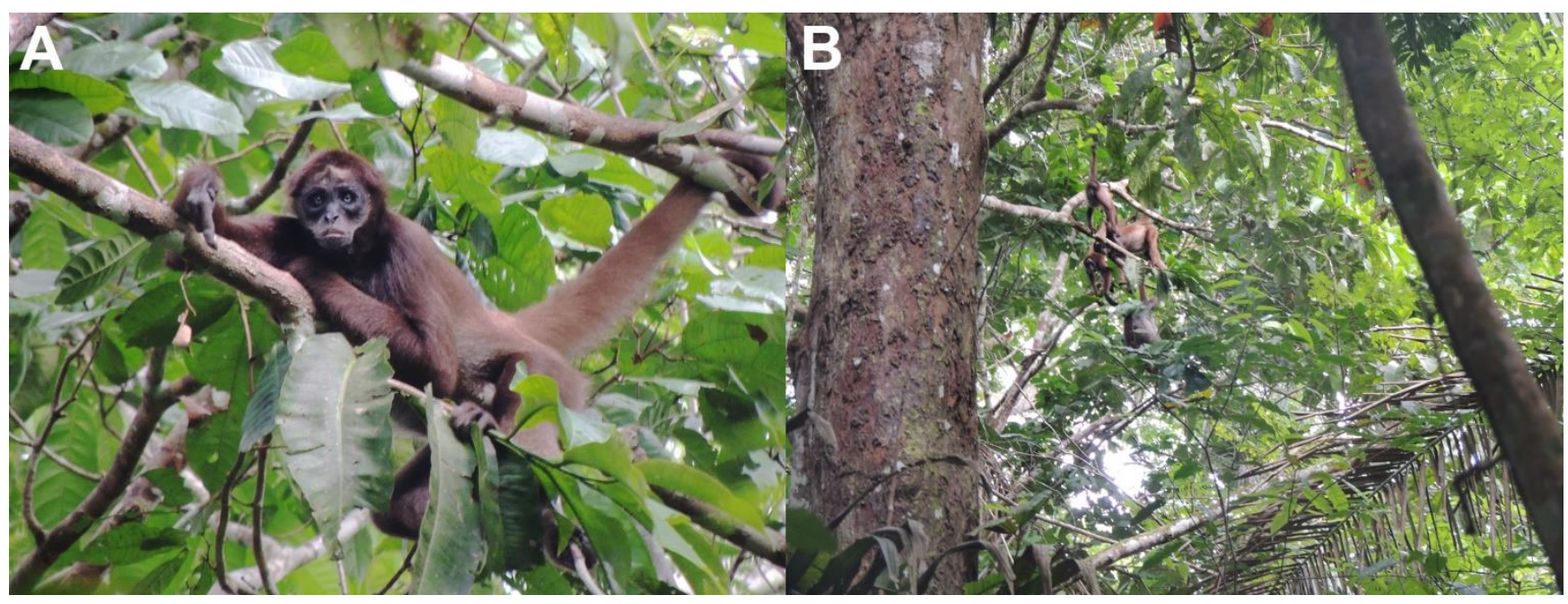

Figura 2. Registro fotográfico de un adulto de Ateles hybridus (A) y del grupo observado (B) en un fragmento de bosque del municipio de Barrancabermeja, Santander, Colombia.

Es importante señalar que este fragmento de bosque, con un tamaño de 384,5 ha, se encuentra aislado y rodeado por una matriz de potreros utilizados para la ganadería bufalina. Si bien la tala y la cacería están prohibidas al interior del bosque, ocasionalmente se realizan talas selectivas de árboles de gran tamaño para el uso doméstico de la finca. Es el único fragmento de bosque en la zona, en donde se encuentra la especie, y aunque existen otros fragmentos boscosos en el área, corresponden a vegetación secundaria alta y franjas de bosque ripario donde no se tuvieron registros de la especie, y los pobladores locales tampoco la han observado. Asimismo se observó que este bosque es el único que provee de zonas con dosel alto y árboles frutales, los cuales requiere la especie para sobrevivir (Aldana et al. 2008).

Este registro, a pesar de encontrarse en el área de distribución esperada de la especie, resalta aún más la importancia de los fragmentos de bosque natural en el Valle del Magdalena Medio, así como la sensibilidad, vulnerabilidad y amenaza de la especie en esta región del país. De esta manera, este reporte busca llamar aún más la atención para la conservación de la especie, para que organizaciones gubernamentales, privadas y sin ánimo de lucro busquen la preservación de estos fragmentos de bosque y la especie, a través de alianzas con los finqueros para disminuir la fragmentación y generar la conectividad en la zona. También es pertinente mencionar que en el municipio de Barrancabermeja en donde el petróleo ha sido parte de la historia social y ambiental de la zona, ha causado que los estudios ambientales en los últimos años sean el único acercamiento para conocer el estado actual de la biodiversidad de la región, estudios que realizados adecuadamente aportan al conocimiento del estado actual de los mamíferos y de otros grupos faunísticos del país.

\section{Agradecimientos}

Agradecemos a las empresas Ingeniería Strycon S.A.S. y Ecopetrol S.A. por el soporte logístico y económico en la realización de las caracterizaciones de fauna silvestre en el área de estudio.

\section{Mammalogy Notes | Notas Mastozoológicas \\ Sociedad Colombiana de Mastozoología Vol. 3 Num. 1| 2016}




\section{Referencias}

ALDANA, A. M., et al. 2008. Habitat characterization and population density of brown spider monkeys (Ateles hybridus) in Magdalena Valley, Colombia. Neotropical Primates 15(2): 46-50.

DEFLER, T. R., et al. 2006. Marimonda del Magdalena Ateles hybridus. Pp. 98-101 En Libro Rojo de los mamíferos de Colombia. (Rodríguez-Mahecha JV, et al. eds.), Conservación Internacional Colombia., Ministerio de Ambiente, vivienda y Desarrollo territorial. Bogotá, Colombia.

LINK, A., et al. 2013. Conservation status in Colombia of one of the most threatened primates: The brown spider monkey (Ateles hybridus). Pp. 87-117 En Primates Colombianos en Peligro de extinción (T. R. Defler, et al. eds.), Asociación Primatológica Colombiana. Bogotá, Colombia.

LINK, A., et al. 2010. Initial effects of fragmentation on the density of three neotropical primate species in two lowland forests of Colombia. Endangered Species Research 13(1): 41-50.

LINK, A., et al. 2012. Diet of the Critically Endangered Brown Spider Monkey (Ateles hybridus) in an Inter-Andean Lowland Rainforest in Colombia. American Journal of Primatology 74(12): 1097-1105.

MADS 2014. Resolución 0192 del 10 de febrero de 2014. "Por la cual se declaran las especies silvestres que se encuentran amenazadas en el territorio nacional y se toman otras determinaciones". República de Colombia. Bogotá D. C., Colombia: Ministerio de Medio Ambiente y Desarrollo Sostenible.

MITTERMEIER, R. A., et al. (eds.). 2012. Primates in Peril: The World's 25 Most Endangered Primates 2012-2014. IUCN/SSC Primate Specialist Group (PSG), International Primatological Society (IPS), Conservation International (CI), and Bristol Conservation and Science Foundation. Bristol, UK, 40.

URBANI, B., et al. 2008. Ateles hybridus. The IUCN Red List of Threatened Species 2008: e.T39961A10280054. Recuperado el 21 de Septiembre de 2015, de http://dx.doi.org/10.2305/IUCN.UK.2008.RLTS.T39961A10280054.en 